\title{
Stage I Uterine Corpus Cancer AJCC v8
}

National Cancer Institute

\section{Source}

National Cancer Institute. Stage I Uterine Corpus Cancer A/CC v8. NCI Thesaurus. Code C139802.

Stage I includes: T1, N0, M0. T1: Uterine corpus carcinoma or carcinosarcoma with tumor confined to the corpus uteri, including endocervical glandular involvement. NO: No regional lymph node metastasis. M0: No distant metastasis. (AJCC 8th Ed.) 